\title{
Borophene Nanoribbon via Strain Engineering for Hydrogen Evolution Reaction: A First-principles Study
}

Xiaoyuan Wang, Rongyao Wu, Pengfei Tian, Yabin Yan*, Yang Gao*, Fuzhen Xuan*

Key Laboratory of Pressure Systems and Safety, Ministry of Education, School of Mechanical Power and Engineering, East China University of Science and Technology, Shanghai, 200237, PR China

Figure S1 shows the atomic structures of nanoribbons of 4 polymorphs of borophene which were experimentally investigated in a very recent study ${ }^{1}$.

(a)

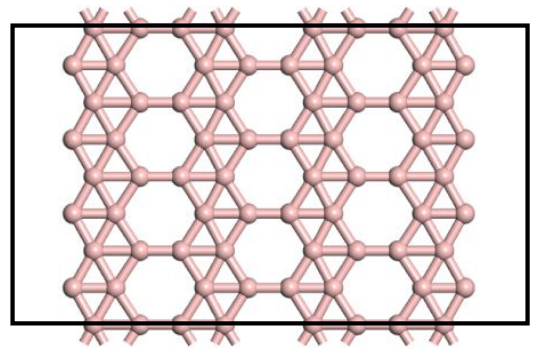

(c)

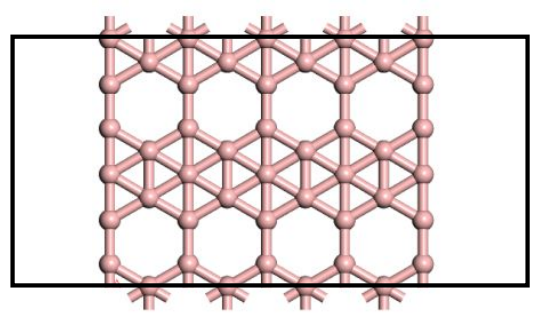

(b)

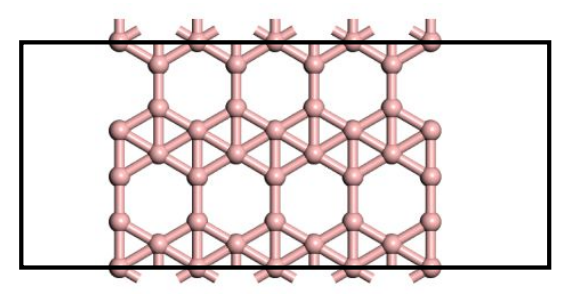

(d)

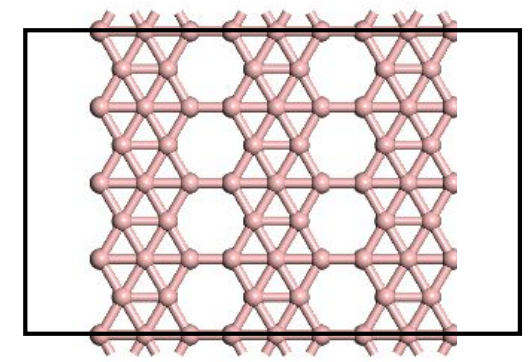

Figure S1 The atomic structures of nanoribbons of 4 polymorphs of borophene ${ }^{1}$. (a) $v_{1 / 5}$ borophene, (b) $v_{1 / 5}-30^{\circ}$ borophene, (c) $v_{1 / 6}$ borophene, and (d) $v_{1 / 6}-30^{\circ}$ borophene.

The $\Delta G_{H}$ of ABNRs with different hydrogen coverage are shown in Figure S2. At a certain hydrogen coverage (1/3), the ABNRs have a significant near-zero $\Delta G_{H}$, indicating the highly active catalysis. It should be noted that, from $1 / 2$ to $1 / 12$ hydrogen coverage, the $\Delta G_{H}$ go up totally and fluctuate a bit, and tend to a level near $0.16 \mathrm{eV}$ under the decreasing hydrogen coverage. The fluctuations may be induced by the interaction hydrogen atoms as fluctuations at high hydrogen coverage are larger than that at low hydrogen coverage, meanwhile, since ABNRs are single layer 2D material, the influence that hydrogen atoms act on ABNRs is another factor that cause the 
fluctuations. ${ }^{2,3}$

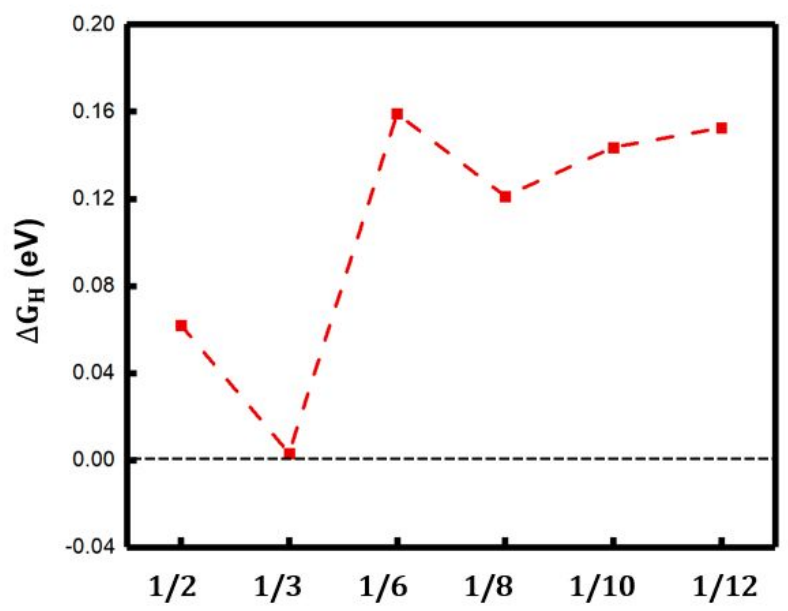

Figure S2 The relation between $\Delta G_{H}$ and hydrogen coverage ranging from $1 / 2$ to $1 / 12$

Figure S3 shows the relaxed structure of 4 7-ABNRs. As the increasing width is reported to have a positive effect that the wider ABNRs are, the more stable the structures become, the 4 7-ABNRs undergo edge reconstruction, which is consistent with the result in reference, in which the buckled edges unfold and the central boron atoms can maintain its structure. It is obvious that the specific numbers of buckled central boron atoms which maintain its atomic order of 2-Pmmn phase gets bigger with the increasing width. In fact, precisely, the number of boron atoms that undergo edge reconstruction remain unchanged (marked by red dashed line). Hence, the conclusion that edge reconstruction has no relation with width can be derived. This edge reconstruction is consistent with previous study. ${ }^{4}$

(a)

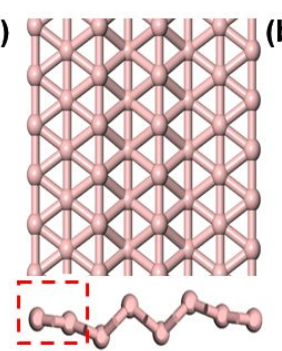

(b)

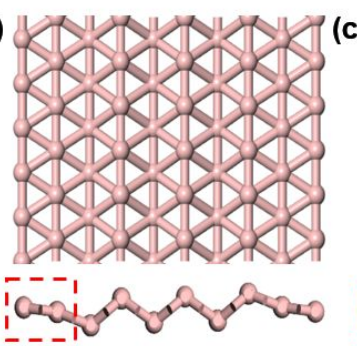

(c)

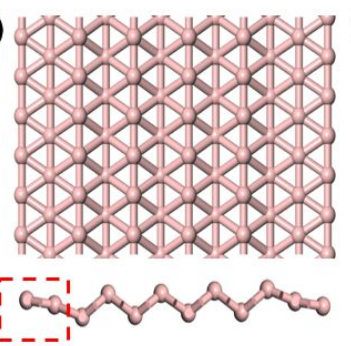

(d)

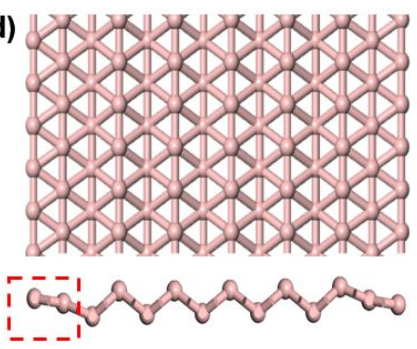

Figure S3 Relaxed structure of 4 7- ABNRs.

The trends of energy and stress with strain is shown in Figure S4, suggesting the elongation at break is beyond $5 \%$ for ABNRs. 

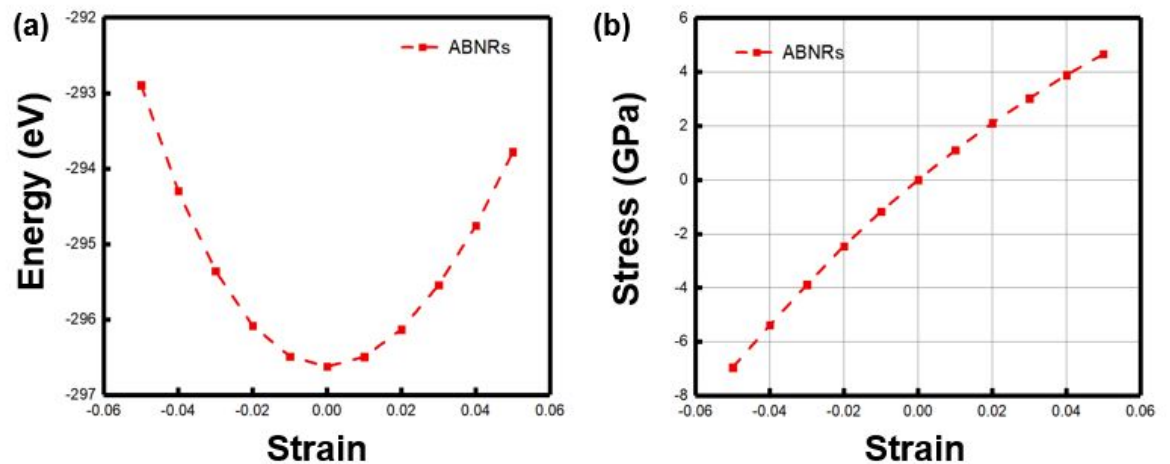

Figure S4 (a) The energy-strain diagram of ABNRs, and (b) the stress-strain diagram of ABNRs.

In Figure S5, the number of electrons corresponding to the filled states up to fermi level of the whole $p$ orbital at $\varepsilon=-4 \%, 0 \%, 2 \%, 5 \%$ is calculated to be $1.320 \mathrm{e}, 1.292 \mathrm{e}, 1.274 \mathrm{e}$, and $1.270 \mathrm{e}$ respectively, which indicates the charge transfer from edge to center.

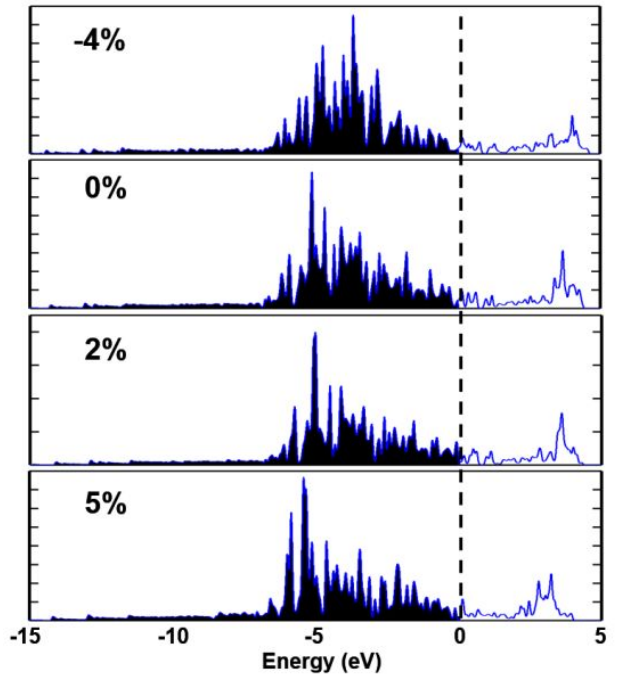

Figure S5 The projected p orbital density of states diagrams for the B atom in 4- ABNRs with different strain magnitudes

In Figure S6, the ELFs of $\varepsilon=-4 \%, 0 \%$, and $5 \%$ show the charge delocalization with the increment of tensile strain in the B-B bonds of ABNRs. When ABNRs is stretched, the localized extra $\pi$ bonds are weakened due to the charge delocalization, and subsequently induces weaker B-H bonds at the edge of ANBRs. 


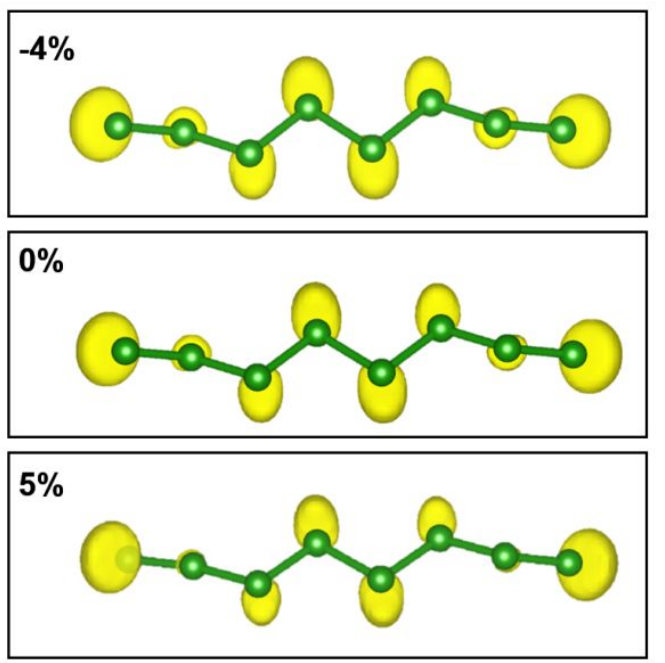

Figure S6 ELF for ABNRs with (a) $-4 \%$, (b) $0 \%$ and (c) 5\% strain.

Table S1 lists the atom coordinates of relaxed structures with different strains, where boron atoms at the intersection of the two lines are denoted by $\mathrm{B}_{i-j}$, and $i$ and $j$ are the numbers of rows and columns, respectively. In addition, due to structural symmetry, only one hydrogen atom and 24 boron atoms in rows 1 to 8 and columns 1 to 3 are shown.

Table S1 Atom coordinates of relaxed structures with different strains.

\begin{tabular}{cccc}
\hline Atom & $-4 \%$ & $-5 \%$ & $5 \%$ \\
\hline $\mathrm{H}$ & $(-0.033,0.104,0.325)$ & $(-0.018,0.374,0.730)$ & $(0.056,-0.020,-0.002)$ \\
$\mathrm{B}_{1-1}$ & $(0.126,0.025,-0.062)$ & $(0.198,0.184,0.112)$ & $(-0.114,0.044,0.145)$ \\
$\mathrm{B}_{1-2}$ & $(0.079,0.049,0.174)$ & $(0.113,0.265,0.540)$ & $(-0.083,0.032,0.127)$ \\
$\mathrm{B}_{1-3}$ & $(-0.033,0.075,0.349)$ & $(-0.017,0.294,0.789)$ & $(0.056,-0.060,0.022)$ \\
$\mathrm{B}_{2-1}$ & $(0.150,0.001,-0.200)$ & $(0.232,0.135,-0.155)$ & $(-0.172,0.034,0.060)$ \\
$\mathrm{B}_{2-2}$ & $(0.116,0.027,0.010)$ & $(0.193,0.211,0.256)$ & $(-0.093,0.029,0.085)$ \\
$\mathrm{B}_{2-3}$ & $(-0.015,0.067,0.354)$ & $(-0.024,0.31,0.862)$ & $(0.007,0.024,0.099)$ \\
$\mathrm{B}_{3-1}$ & $(0.097,-0.012,-0.100)$ & $(0.197,0.118,-0.111)$ & $(-0.112,0.037,-0.197)$ \\
$\mathrm{B}_{3-2}$ & $(0.009,0.064,0.215)$ & $(0.002,0.322,0.556)$ & $(-0.028,0.011,-0.186)$ \\
$\mathrm{B}_{3-3}$ & $(-0.033,0.027,0.225)$ & $(-0.017,0.294,0.426)$ & $(0.055,0.027,-0.191)$ \\
$\mathrm{B}_{4-1}$ & $(0.141,-0.004,-0.091)$ & $(0.213,0.066,0.023)$ & $(-0.177,-0.026,0.007)$ \\
$\mathrm{B}_{4-2}$ & $(0.085,-0.042,-0.083)$ & $(0.167,-0.047,0.071)$ & $(-0.059,-0.023,0.158)$
\end{tabular}




\begin{tabular}{lccc}
$\mathrm{B}_{4-3}$ & $(0.003,0.072,0.113)$ & $(-0.005,0.320,0.577)$ & $(0.030,-0.021,-0.057)$ \\
$\mathrm{B}_{5-1}$ & $(0.098,0.064,0.041)$ & $(0.174,0.168,0.140)$ & $(-0.127,0.001,-0.021)$ \\
$\mathrm{B}_{5-2}$ & $(0.033,0.005,0.023)$ & $(0.149,0.047,0.226)$ & $(-0.008,0.025,0.034)$ \\
$\mathrm{B}_{5-3}$ & $(-0.033,0.081,0.108)$ & $(-0.008,0.476,0.586)$ & $(0.055,0.003,0.042)$ \\
$\mathrm{B}_{6-1}$ & $(0.128,0.003,0.084)$ & $(0.182,0.059,-14.300)$ & $(-0.156,-0.015,0.129)$ \\
$\mathrm{B}_{6-2}$ & $(0.062,0.001,0.070)$ & $(0.142,0.025,-14.230)$ & $(-0.064,-0.019,-14.642)$ \\
$\mathrm{B}_{6-3}$ & $(0.001,0.018,0.019)$ & $(-0.002,0.016,0.041)$ & $(0.009,-0.015,0.142)$ \\
$\mathrm{B}_{7-1}$ & $(0.113,0.027,0.211)$ & $(0.207,0.087,0.675)$ & $(-0.119,-0.004,-0.042)$ \\
$\mathrm{B}_{7-2}$ & $(0.027,-0.003,-0.083)$ & $(0.011,0.005,-0.006)$ & $(-0.021,-0.009,0.136)$ \\
$\mathrm{B}_{7-3}$ & $(-0.033,0.017,0.077)$ & $(-0.020,0.009,-0.004)$ & $(0.056,-0.018,-0.073)$ \\
$\mathrm{B}_{8-1}$ & $(0.132,0.019,0.19)$ & $(0.198,0.112,0.737)$ & $(-0.152,-0.001,-0.012)$ \\
$\mathrm{B}_{8-2}$ & $(0.064,0.002,0.083)$ & $(0.079,0.019,0.306)$ & $(-0.058,-0.01,0.008)$ \\
$\mathrm{B}_{8-3}$ & $(0,0,0)$ & $(0,0,0)$ & $(0,0,0)$ \\
\hline
\end{tabular}

\section{Reference}

(1) Li, Q.; Kolluru, V. S. C.; Rahn, M. S.; Schwenker, E.; Li, S.; Hennig, R. G.; Darancet, P.; Chan, M. K. Y.; Hersam, M. C. Science 2021, 371, 1143-1148.

(2) Shi, L.; Ling, C. Y.; Ouyang, Y. X.; Wang, J. L. Nanoscale 2017, 9, 533.

(3) Chen, Y. L.; Yu, G. T.; Chen, W.; Liu, Y. P.; Li, G. D.; Zhu, P. W.; Tao, Q.; Li, Q. J.; Liu, J. W.; Shen, X. P.; Li, H.; Huang, X. R.; Wang, D. J.; Asefa, T.; Zou, X. J. Am. Chem. Soc. 2017, 139, 12370 .

(4) Liu, Y. X.; Dong, Y. J.; Tang, Z. Y.; Wang, X. F.; Wang, L.; Hou, T. J.; Lin, H. P.; Li, Y. Y. J. Mater. Chem. C 2016, 4, 6380. 